Tanja A. Börzel

\title{
Europäische Integrationstheorie - nicht obsolet, aber reformbedürftig
}

\section{1. „Der Europäische Verfassungsentwurf ist tot « ${ }^{1}$ - lang lebe die Europäische Verfassung!}

Das französische und niederländische Nein hat dem Europäischen Verfassungsvertrag einen empfindlichen Schlag versetzt; aber ob er wirklich tödlich war, bleibt abzuwarten und wird von den (innen)politischen Entwicklungen der nächsten Monate abhängen. Auch scheinen die Totengräber des Verfassungsvertrages zu vergessen, dass Europa bereits eine Verfassung hat, die von dem in den Europäischen Verträgen niedergelegten Primärrecht der Europäischen Union gebildet wird (Joerges 2003; Stone Sweet/Sandholtz 1998). Die Nachricht sowohl vom Tod der Europäischen Verfassung wie auch der Europäischen Integrationstheorie könnte also verfrüht sein.

Die ablehnenden Referenden in Frankreich und den Niederlanden haben ohne Frage eine politische Krise in der EU ausgelöst, die durch die kurz darauf gescheiterten EU-Haushaltsverhandlungen noch verstärkt wurde. Ob diese Krise jedoch den Integrationsprozess grundsätzlich infrage stellt oder gar das Scheitern des gesamten Projekts Europa bedeuten könnte, ist eine empirische Frage, die sich zu diesem Zeitpunkt noch nicht abschließend klären lässt. Der Ratifikationsprozess ist noch nicht abgeschlossen. Und selbst wenn die Mitgliedsstaaten sich entschließen sollten, den Verfassungsvertrag gänzlich aufzugeben, gibt es eventuell noch andere Möglichkeiten, die im Verfassungsvertrag angestrebten Reformen durchzuführen und Effektivität und Legitimität einer erweiterten EU zu erhöhen (Jopp/Kuhle 2005; Maurer 2005).

Die europäische Politik hat sich erst einmal eine Denkpause verordnet, welche die Politikwissenschaft kritisch und beratend begleiten sollte. Aus integrationstheoretischer Sicht stellt sich die Situation jedoch weniger dramatisch dar. Trotz der politischen Krise ist die EU von der »Eurosklerosis« der 1970er Jahre (noch) weit entfernt. Lebte Ernst Haas noch, so würde er zunächst feststellen, dass sich die Mitgliedsstaaten mit dem Verfassungsvertrag auf einen bereits in Maastricht angestrebten Integrationsfortschritt geeinigt haben, an dem zwei Regierungskonferenzen (Amsterdam und Nizza) zuvor gescheitert waren. Dem Verfassungskonvent gelang es, die berühmten left-overs von Maastricht endlich abzuarbeiten. Die institutionellen Reformen steigern nicht nur die Handlungsfähigkeit einer erweiterten EU. Die

1 Beck/Giddens (2005: 13). 
Konventsmethode oder die stärkere Einbeziehung der nationalen Parlamente in den Politikprozess der EU leisten darüber hinaus einen wichtigen Beitrag zur demokratischen Legitimation des europäischen Mehrebenensystems. Sollte dieser Integrationserfolg nun tatsächlich an der Ratifizierung durch die Mitgliedsstaaten scheitern, stellt das die Europapolitik vor ein Dilemma, da weder der traditionelle Weg der Regierungskonferenz noch die alternative Konventsmethode in der Lage sind, die für die Effektivität und Legitimität der EU notwendigen Reformen zu erwirken. Für die Europäische Integrationstheorie ergibt sich hingegen ein Forschungsproblem, mit dem sie sich die letzten 50 Jahre nicht auseinander setzen musste, dem sie sich aber - wie ich im Folgenden argumentieren werde - stellen muss, wenn sie nicht wirklich obsolet werden will.

\section{Vom Ende der »big bargains«}

Seit 1954 die Europäische Politische Gemeinschaft in der französischen Nationalversammlung gescheitert war, beschäftigten sich zunächst der Neofunktionalismus und später dann auch der (Liberale) Intergouvernementalismus mit den politischen Entscheidungen zur Schaffung bzw. Änderung der Europäischen Verträge. Während sich die zwei großen Integrationsschulen über die Rolle der nationalen Regierungen und der supranationalen Akteure uneinig waren, konzentrierten sich beide auf die großen Regierungskonferenzen von 1957, 1986, 1990, 1997 und 2000, bei denen die Mitgliedsstaaten als Herren der Verträge das Primärrecht schufen, das heute die Europäische Verfassung ausmacht. Das führte in gewisser Weise zu einem »Selektionsfehler « auf Seiten der abhängigen Variable, da nahezu ausschließlich die Erfolgsfälle der europäischen Integration untersucht worden sind. ${ }^{2}$ Lediglich Ernst Haas beschäftigte sich mit der Eurosklerose, welche 1965 durch die Krise der »Politik des leeren Stuhls« eingeleitet worden war und kaum mit den Annahmen seiner neofunktionalistischen Theorie vereinbar schien. Enttäuscht von der Hartnäckigkeit national(istisch)er Politik erklärte er 1975 die regionale Integrationstheorie schließlich für obsolet (Haas 1975). Dies geschah jedoch vor allem aufgrund gescheiterter Versuche der nationalen Regierungen, den Integrationsprozess selbst im Bereich der low politics (Landwirtschaft, Vier Freiheiten) voranzubringen. Das zentrale Problem war die fehlende Bereitschaft der Mitgliedsstaaten, sich auf EU-Ebene auf weitere Reformen zu einigen. Die Frage der Ratifikation der Vertragsreformen fand hingegen kaum Beachtung. Daran änderten auch das dänische Nein zum Maastrichter Vertrag und das irische Nein zum Vertrag von Nizza nichts, das Problem ließ sich schließlich durch Nachverhandlungen beheben.

Diesmal scheint alles anders. Zum ersten Mal seit mehr als 50 Jahren steht das Inkrafttreten einer weit reichenden Vertragsänderung ernsthaft in Frage. Nicht nur haben sich mit Frankreich und den Niederlanden zwei Gründerstaaten der EG dem

2 So beginnt z. B. Andrew Moravcsik (1998) seine Analyse der big bargains in seinem Buch »The Choice for Europe « erst mit den Römischen Verträgen. 
Integrationsfortschritt in den Weg gestellt. Anders als in Dänemark und Irland sind die ablehnenden Voten sehr deutlich ausgefallen und bergen die Gefahr eines negativen »Kaskadeneffektes« für die Mitgliedsstaaten, die wie Großbritannien, Dänemark, Irland, Portugal, Polen und Tschechien ein Referendum noch vor sich haben. Dass die Integrationstheorien diese Entwicklung nicht antizipiert haben, ist nicht weiter verwunderlich, aber auch nicht wirklich problematisch. Die Frage ist vielmehr, ob sie gegebenenfalls in der Lage sind, das Scheitern des Verfassungsvertrages im Ratifikationsprozess zu erklären. Jede Theorie muss sich daran messen lassen, ob sie die Varianz auf ihrer zentralen Untersuchungsvariable erfassen kann. Integrationstheorien sind deshalb nicht nur gefordert, Integrationsfortschritt $\mathrm{zu}$ erklären, sondern auch Stagnation und Rückschritt - und zwar unabhängig davon, in welcher Phase die Krise auftritt. Die Frage ist also nicht so sehr, ob die Integrationstheorien obsolet geworden sind, sondern ob sie ausbaufähig genug sind, um den Ratifikationsprozess von Vertragsänderungen zu erfassen und damit nicht obsolet zu werden.

Im Folgenden werde ich erörtern, inwieweit die Annahmen der beiden klassischen Integrationstheorien, des Liberalen Intergouvernementalismus und des Neofunktionalismus, erweitert werden können, damit sie nicht nur die Entstehung des Verfassungsvertrags, sondern auch sein eventuelles Scheitern im Ratifikationsprozess erklären können. Obwohl sich mittlerweile die Integrationsforschung theoretisch ausdifferenziert hat (vgl. Diez/Wiener 2003; Bieling/Lerch 2005), sind die beiden Ansätze in ihren (konstruktivistischen) Spielarten nach wie vor dominierend, zumal hier nur die Hauptargumentationslinien angesprochen werden.

\section{Der Liberale Intergouvernementalismus: Zurück zur Zwei-Ebenen-Perspektive}

Der Liberale Intergouvernementalismus geht davon aus, dass Staaten die zentralen Akteure im Integrationsprozess sind. Integrationsfortschritt kann es nur dann geben, wenn die verhandlungsstarken Staaten ein gemeinsames Interesse verfolgen, dass sie auf der europäischen Ebene am besten realisiert sehen. Während der Liberale Intergouvernementalismus zu den staatszentrierten Ansätzen der Integrationsforschung zählt, behandelt er Staaten nicht als einheitliche Akteure. Vielmehr geht er davon aus, dass innerstaatliche Interessen die von den nationalen Regierungen im europäischen Verhandlungsprozess vertretenen Positionen maßgeblich beeinflussen (vgl. Moravcsik 1993, 1998).

Der Liberale Intergouvernementalismus verlegt sich auf die Erklärung von Integrationsfortschritt und vernachlässigt dabei gescheiterte Integrationsversuche wie die Europäische Politische Gemeinschaft 1953 oder den Werner-Plan 1974. Auch interessiert er sich nicht für die Umsetzung von den auf Regierungskonferenzen ausgehandelten Vertragsreformen. Trotzdem sollte er in der Lage sein, eine gescheiterte Ratifikation zu erklären, da der Liberale Intergouvernementalismus auf dem ZweiEbenen-Ansatz aufbaut, für den das Problem der involuntary defection eine zentrale Rolle spielt (Putnam 1988). Er trifft allerdings bestimmte Annahmen über die Natur 
des europäischen Mehrebenensystems, die der Zwei-Ebenen-Ansatz so nicht teilt und die auch nicht für jedes internationale Verhandlungssystem als gegeben angesehen werden können (ob sie für die EU zutreffen, kann hier nicht weiter diskutiert werden): Zum einen gilt der Informationsgrad der Regierungen hinsichtlich der eigenen Präferenzen, der Präferenzen der anderen Regierungen sowie der Handlungsoptionen als verhältnismäßig hoch. Zum anderen werden die Transaktionsund Informationskosten in europäischen Verhandlungen als gering veranschlagt, d. h. die Verhandlungen sind natürlich effizient. Jeder Staat kann seine Interessen gemäß seiner Verhandlungsmacht durchsetzen. Das Ergebnis ist also pareto-optimal, und es besteht kein Anreiz für Regierungen und innerstaatliche Interessen zu »defektionieren«, zumal Nachverhandlungen wenig Aussicht auf Erfolg haben. Deshalb erscheint die Nichtratifikation eines einmal erzielten Verhandlungsergebnisses unwahrscheinlich bzw. kann aufgrund der getroffenen Annahmen vom Liberalen Intergouvernementalismus nicht erklärt werden.

Der Zwei-Ebenen-Ansatz, wie er von Robert Putnam für internationale Verhandlungssysteme entwickelt wurde, geht hingegen nicht davon aus, dass internationale Verhandlungen natürlich effizient sind (Putnam 1988; Evans et al. 1993). Es können empfindliche Informationsasymmetrien zwischen Staaten bestehen; die Kosten der Informationsbeschaffung sind nicht notwendigerweise immer niedrig und der generelle Informationsgrad muss nicht hoch sein. Deshalb kann es auch zum Problem der involuntary defection kommen - Regierungen sind bereit, sich an internationale Verträge zu binden, die auf internationaler Ebene getroffene Einigung scheitert jedoch an den fehlenden Mehrheiten im innenpolitischen Ratifikationsprozess. Dies tritt vor allem dann ein, wenn die Regierung die Größe des innerstaatlichen win sets, also der ratifikationsfähigen Verhandlungsergebnisse, überschätzt hat, weil der Aggregationsprozess innenpolitischer Interessen unvollständig war, also nicht alle relevanten Akteure berücksichtigt wurden. Neben dem Problem der unvollständigen Information kann die Regierung auch versucht haben, innerstaatliche Informationsasymmetrien auszunutzen, um über die europäische Ebene die Durchsetzung von politischen Projekten zu legitimieren, für die es innenpolitisch sonst keine Mehrheiten gegeben hätte (strategische Selbstbindung, cutting slack). Schließlich können innerstaatliche Akteure die Durchsetzungsfähigkeit der eigenen Regierung in internationalen Verhandlungen überschätzt haben und tragen deshalb erzielte Kompromisslösungen (Koppelgeschäfte, Paketlösungen, Ausgleichszahlungen) nicht mit.

Da die französische und niederländische Ratifikation des Verfassungsvertrags in Referenden gescheitert ist, scheint es durchaus plausibel, dass die Regierungen falsche Annahmen über die Wählerpräferenzen getroffen hatten. In beiden Ländern wäre schließlich eine parlamentarische Ratifikation ausreichend gewesen. Eine mögliche Erklärung für die Fehleinschätzung könnte darin liegen, dass die innerstaatliche Interessenaggregation im Vorfeld der Regierungskonferenz auf dem üblichen Wege über Parteien und Interessengruppen erfolgte, während in den Referenden der direkte Wählerwille zum Ausdruck kam.

Gerade weil der Zwei-Ebenen-Ansatz ohne die von Andrew Moravcsik getroffenen Annahmen über die Natur des europäischen Mehrebenenverhandlungssystems 
auskommt, ist er in der Lage, die gescheiterte Ratifikation von internationalen Verhandlungen grundsätzlich zu erklären. Das Problem, das sich für Wissenschaft und Politik gleichermaßen stellt, ist die Bestimmung des tatsächlichen win sets: Wie kann der Wählerwille erfasst werden, wenn die innenpolitische Interessenaggregation nicht über intermediäre Instanzen erfolgen kann, weil die Ratifikation nicht über repräsentative Institutionen erfolgt?

\section{Der Neofunktionalismus: Vom »permissive consensus« zum »constraining dissensus ${ }^{3}$}

Auch der Neofunktionalismus bezieht die innerstaatliche Ebene in die Erklärung des Integrationsprozesses mit ein. Anders als im Liberalen Intergouvernementalismus üben innerstaatliche Akteure allerdings ihren Einfluss weniger über ihre Regierungen aus, sondern nutzen die Europäische Kommission und den Europäischen Gerichtshof, um ihre Interessen in den Integrationsprozess einzubringen. Die strategische Allianz zwischen gesellschaftlichen Interessen und supranationalen Akteuren führt über Prozesse des funktionalen spillovers zu einer Ausweitung der Integration, indem die erfolgreiche grenzüberschreitende Kooperation in einem Sachbereich auf andere Sachbereiche ausgedehnt wird. Die fortschreitende Integration führt zu einer Verlagerung der politischen Loyalitäten und Erwartungshaltungen der gesellschaftlichen und wirtschaftlichen Eliten auf die europäische Ebene (Haas 1958, 1964; Lindberg/Scheingold 1970). Dieser politische spillover geht mit einer steigenden Konflikthaftigkeit der gemeinsamen Entscheidungsfindung in europäischen Institutionen einher und führt zu einer Ausweitung der beteiligten und interessierten Akteure. Der Ratifikationsprozess in Frankreich, den Niederlanden und anderen Mitgliedsstaaten zeugt von dieser zunehmenden Politisierung der europäischen Integration. Ironischerweise scheint sich jedoch die vom Neofunktionalismus damit verknüpfte Erwartung einer stetigen Vertiefung (Haas 1958: 526f; Lindberg 1963) nicht zu erfüllen. Während Meinungsumfragen auf einen Loyalitätstransfer nationaler Eliten schließen lassen, ist die Unterstützung der breiten Öffentlichkeit über die letzten Jahre stagniert und in einigen Mitgliedsstaaten sogar zurückgegangen (Eichenberg/Dalton 1993; Hooghe/Marks 2005).

Die Referenden in Frankreich und den Niederlanden haben den Bürgerinnen und Bürgern die Gelegenheit gegeben, ihren Unmut und ihre Ängste gegenüber der europäischen Integration unmittelbar zu artikulieren. Sie haben die politische Auseinandersetzung aus den für den Neofunktionalismus zentralen intermediären Instanzen herausgetragen. Damit nimmt die Politisierung der Integration Formen an, die sich neofunktionalistisch nur schwer theoretisch rückbinden lässt. Bisher war die öffentliche Meinung für den Neofunktionalismus keine relevante Größe; der permissive consensus (Lindberg/Scheingold 1970: 62) bildete eine nicht näher hinterfragte Kontextbedingung für das Fortschreiten der Integration, die jetzt nicht mehr als

3 Hooghe/Marks (2005: 5). 
gegeben gelten kann. Dies ist vor allem dann problematisch, wenn Referenden als Instrument zur Ratifizierung von Vertragsreformen eingesetzt werden.

Die französische und niederländische Ablehnung des Verfassungsvertrags bedeutet nicht notwendigerweise ein Nein zu Europa, weil sich politische Loyalitäten wie in den 1970er Jahren weiterhin auf den Nationalstaat richten. Aus neofunktionalistischer Perspektive ließe sich vielmehr auch argumentieren, dass die Bürgerinnen und Bürger vielleicht nicht ihre Loyalitäten, aber doch ihre politischen Erwartungen zunehmend auf die europäische Ebene übertragen, die Europäische Union jedoch immer weniger in der Lage ist, diese Erwartungen zu erfüllen. Paradoxerweise hätten dann die Franzosen und Niederländer genau die Reformen verhindert, die notwendig wären, um die Handlungsfähigkeit der europäischen Institutionen zu stärken, damit diese die gestiegenen Erwartungen erfüllen können.

\section{Schlussbetrachtungen}

Selbst wenn der Europäische Verfassungsvertrag letztendlich scheitern sollte, hat die Europäische Integrationstheorie keineswegs wieder versagt. Sie steht vielmehr vor einer neuen Herausforderung, der sie sich stellen muss, wenn sie in Zukunft nicht obsolet werden will. Dies gilt insbesondere dann, wenn die Bedeutung von Referenden in der Europapolitik weiter zunehmen sollte. Dabei erweisen sich Referenden für die Politik wie auch für die Wissenschaft als Fluch und Segen zugleich. Einerseits bewirken sie über die zunehmende Politisierung eine Normalisierung der Europapolitik. Die Kontroverse um das »soziale Europa« zeigt, dass die EU endlich in der Tagespolitik angekommen ist; es geht also immer weniger um das »Für« oder »Gegen« Europa, sondern um die Frage, welches Europa zunehmend die politischen Debatten bestimmt. Die Franzosen haben nicht »Non« zu Europa gesagt; sie wollen vielmehr ein anderes Europa (Maurer 2005; von Oppeln 2005). Damit gewinnt die politische Finalität eine neue Form, die sehr viel eher an traditionelle Konfliktlinien der Innenpolitik anschlussfähig ist als an die Auseinandersetzung um die Staatlichkeit der EU. Die Politisierung birgt nicht nur neue Chancen für die Legitimation der EU. Sie öffnet die EU auch (noch) weiter für Theorien und Konzepte aus der Vergleichenden Regierungslehre und der policy-Analyse. Andererseits lösen Referenden den europapolitischen Diskurs, sofern er bisher stattgefunden hat, aus den intermediären Institutionen (Parteien, Interessenverbände) und verlagern ihn in den öffentlichen Raum, wo er sich institutionell nur schwer wieder einfangen lässt. Dies stellt nicht nur die Politik vor neue Herausforderungen. Sowohl der Liberale Intergouvernementalismus als auch der Neofunktionalismus basieren letztendlich auf Vorstellungen repräsentativer Demokratie, die beim Neofunktionalismus stark assoziative Züge aufweisen. Letztendlich wird die europäische Integration zwischen (trans)national organisierten Eliten ausgehandelt (siehe auch den Beitrag von Jürgen Neyer in dieser Ausgabe, Seiten 377-382), weshalb sich die beiden Theorien mit plebiszitären Elementen der Politik schwer tun. Sollten sich Referenden tatsächlich zu einem gängigen Instrument der Ratifikation konstitutioneller Reformen in der EU 
entwickeln, könnten Theorien aus dem Bereich der Innenpolitik und der Komparatistik, welche die Öffentlichkeit stärker in den Blick nehmen, den Liberalen Intergouvernementalismus und den Neofunktionalismus vielleicht nicht obsolet machen, aber doch von ihrer nach wie vor dominanten Position in der Integrationsforschung verdrängen.

\section{Literatur}

Bieling, Hans-Jürgen/Lerch, Marika (Hrsg.) 2005: Theorien der europäischen Integration, Wiesbaden.

Beck, Ulrich/Giddens, Anthony 2005: Die Zukunft der EU und ihrer Nationen. Ein Aufruf zur europaweiten Diskussion, in: Süddeutsche Zeitung, 1.10.2005, 13.

Diez, Thomas/Wiener, Antje (Hrsg.) 2003: European Integration Theory, Oxford.

Eichenberg, Richard C./Dalton, Russel J. 1993: Europeans and the European Community: The Dynamics of Public Support for European Integration, in: International Organization 47: $3,507-534$.

Evans, Peter B./Jacobson, Harold K./Putnam, Robert D. (Hrsg.) 1993: Double-Edged Diplomacy: International Bargaining and Domestic Politics, Berkeley, CA.

Haas, Ernst B. 1958: The Uniting of Europe: Political, Social, and Economic Forces 19501957, Stanford, CA.

Haas, Ernst B. 1964: Beyond the Nation-State: Functionalism and International Organization, Stanford, CA.

Haas, Ernst B. 1975: The Obsolescence of Regional Integration Theory, Berkeley, CA.

Hooghe, Lisbet/Marks, Gary 2005: The Neofunctionalists Were (almost) Right: Politicization and European Integration, in: Constitutionalism Webpapers (Con WEB) 6: 5 (www.qub.ac.uk/pisp/Research/PaperSeries/ConWEBPapers/; 12.10.2005).

Joerges, Christian 2003: Recht, Wirtschaft und Politik im Prozess der Konstitutionalisierung Europas, in: Jachtenfuchs, Markus/Kohler-Koch, Beate (Hrsg.): Europäische Integration, Opladen, 183-218.

Jopp, Mathias/Kuhle, Gesa S. 2005: Wege aus der Verfassungskrise. Die EU nach den gescheiterten Referenden in Frankreich und den Niederlanden, in: Integration 28: 3, 257-261.

Lindberg, Leon N. 1963: The Political Dynamics of European Economic Integration, Stanford, $\mathrm{CA}$.

Lindberg, Leon N./Scheingold, Stuart A. 1970: Europe's Would-be Polity: Patterns of Change in the European Community, Englewood Cliffs, NJ.

Maurer, Andreas 2005: Die Ratifikationsverfahren zum EU-Verfassungsvertrag. Wege aus der Krise (SWP-Diskussionspapier 8/2005), Berlin.

Moravcsik, Andrew 1993: Preferences and Power in the European Community: A Liberal Intergovernmentalist Approach, in: Journal of Common Market Studies 31: 4, 473-524.

Moravcsik, Andrew 1998: The Choice for Europe: Social Purpose and State Power From Rome to Maastricht, Ithaca, NY.

Putnam, Robert 1988: Diplomacy and Domestic Politics: The Logic of Two-level Games, in: International Organization 42: 2, 427-460.

Stone Sweet, Alec/Sandholtz, Wayne 1998: Integration, Supranational Governance, and the Institutionalization of the European Polity, in: Stone Sweet, Alec/Sandholtz, Wayne (Hrsg.): European Integration and Supranational Governance, Oxford, 1-26.

von Oppeln, Sabine 2005: Das Projekt Europa im Spiegel der französischen Debatte über den europäischen Verfassungsvertrag, in: Dokumente 61: 3, 12-18. 